\title{
Cardiovascular Biomechanics and Biofluids: A Special Issue with a Focus on Modeling of Cardiovascular Structures
}

Cardiovascular biomechanics and biofluids encompass the study of structure and function of biological systems involved in the human circulatory system. The fields have produced a fundamental understanding of the interaction between blood flow and cardiovascular structures, as well as technologies for the surgical and endovascular repair of cardiovascular pathologies. They have provided major advances in biomedical engineering research through the development of bench-top, animal and clinical image-based models for risk assessment of cardiovascular disease, surgical planning, and design and optimization of endovascular devices for interventional applications. A few examples of such applications are heart valve replacement, aneurysm repair, intracranial and coronary artery recanalization, thrombectomy, etc. Many of these research activities are interdisciplinary, involving bioengineers in collaboration with physicians and basic scientists from academia and the medical device industry.

While tremendous advances have been achieved in the management of cardiovascular patients in the last few decades, diseases affecting the cardiovascular system are still the most deadly in the developed world. Biomechanics studies integrate computer modeling with mechanical, fluid mechanical and analytical techniques providing a platform for obtaining more information on a specific disease, which can lead the way for personalized engineering-based diagnostic and treatment planning tools. The need for integrating physical and engineering sciences with the life sciences to advance medical care is recognized by both the National Institutes of Health and the American Heart Association, and is reflected in both organizations' current funding opportunities.

The goal of this special issue is to highlight advances in the field of biomedical engineering that involve basic and applied biomechanics and biofluids. As such, the Annals of Biomedical Engineering is an ideal platform for communicating these advances and state of the art methodologies. The nineteen research articles in this issue address several different pathologies and cardiovascular modeling strategies, comprising ten review articles and nine original research articles. Healthy and diseased biomechanical murine models are discussed in detail by Humphrey and colleagues. The biomechanics of aortic valves and its relationship to biological mechanisms are considered by the Yoganathan group. The pathophysiology of cerebral aneurysms was studied by Lieber et al., while the Cebral laboratory investigated the interrelationships among their risk factors, Watton et al. the role of hemodynamics on their initial growth, and $\mathrm{Lu}$ et al. present an approach for calculating their stresses without specifying wall material properties. Chesler and associates present their work on hemodynamic coupling of the right ventricle with the main pulmonary artery. The fundamentals and applications of buckling phenomena in arteries are described extensively by the Han laboratory. Contributions to the modeling of vascular networks and pathologies are represented by the works of Meng et al. as it relates to the role of wall shear stress, and Taelman et al. concerning multiscale modeling approaches. Coronary artery biomechanics is emphasized

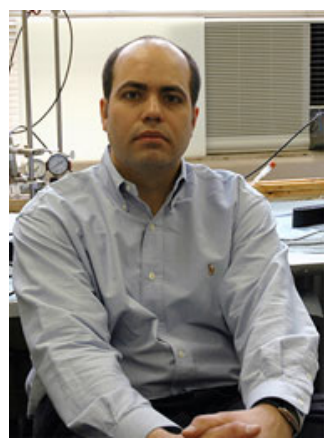

Ender A. Finol.

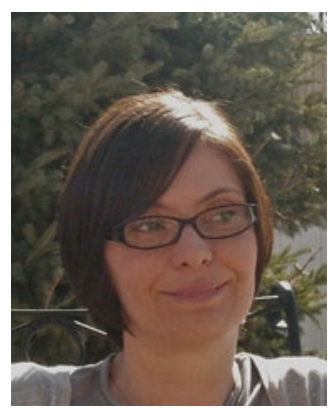

Elena S. Di Martino.

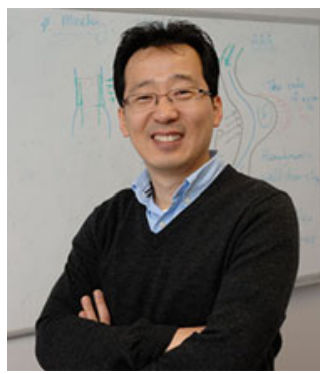

Seungik Baek. by the Vande Geest group regarding the quantification of ex vivo tissue properties, while modeling techniques for coronary stents is provided by the Migliavacca group. The Di Martino laboratory contributed work on the local mechanical characterization of tissues from healthy human atria using a new structural constitutive model. Modeling of abdominal aortic aneurysms is represented in this issue as it relates to the calculation of residual strains, by Polzer et al., the quantification of their failure properties, by Gasser et al., and a critical analysis of the 
relative importance of geometric and biomechanical risk factors, by the Finol group. Vascular biomechanics received consideration in the issue contributed by the works of the Zhang laboratory on biaxial behavior of the porcine aorta, the Rachev group on arterial remodeling of arteries subject to hypertensive conditions, and Swillens et al. on the use of pulse wave velocity to assess vascular material properties noninvasively.

We hope you will find these research articles informative, thought-provoking and timely in highlighting current research in Cardiovascular Biomechanics and Biofluids. We also wish to thank all the reviewers who carefully read these articles and provided their extensive critiques, thereby contributing to the overall quality of the Special Issue.
ENDER A. FinOL

Department of Biomedical Engineering, The University of Texas at San Antonio, San Antonio, TX, USA

Electronic mail: ender.finol@utsa.edu

Elena S. Di Martino

Centre for Bioengineering Research and Education, Department of Civil Engineering, University of Calgary, Calgary, AB, Canada

Electronic mail: edimarti@ucalgary.ca

Seungik Baek

Department of Mechanical Engineering, Michigan State University, East Lansing, MI, USA

Electronic mail: sbaek@egr.msu.edu 